\title{
Circulating miRNAs as Predictor Markers for Activation of Hepatic Stellate Cells and Progression of HCV-Induced Liver Fibrosis
}

\author{
Eman El-Ahwany ${ }^{1}$, Faten Nagy ${ }^{1}$, Mona Zoheiry ${ }^{1}$, Mohamed Shemis ${ }^{2}$, Mona Nosseir ${ }^{3}$, Hoda Abu Taleb ${ }^{4}$, Maged \\ El Ghannam ${ }^{5}$, Rafaat Atta ${ }^{5}$, Suher Zada ${ }^{6}$
}

\footnotetext{
${ }^{1}$ Immunology Department, Theodor Bilharz Research Institute, Giza, Egypt

${ }^{2}$ Biochemistry Department, Theodor Bilharz Research Institute, Giza, Egypt

${ }^{3}$ Pathology Department, Theodor Bilharz Research Institute, Giza, Egypt

${ }^{4}$ Environmental Research Department, Theodor Bilharz Research Institute, Giza, Egypt

${ }^{5}$ Gastroenterology Department, Theodor Bilharz Research Institute, Giza, Egypt

${ }^{6}$ Biology Department, American University in Cairo, Cairo, Egypt
}

\section{Type of article: Original}

\begin{abstract}
Introduction: Liver fibrosis is the excessive accumulation of extracellular matrix that occurs by activation of hepatic stellate cells (HSCs), which has been identified as the major driver of liver fibrosis. Several studies confirmed that miRNAs have regulatory effects on the activation of HSCs by affecting the signaling pathways. The aim of this study was to develop non-invasive diagnostic markers by measuring different circulating miRNAs in serum as predictor markers for early diagnosis of liver fibrosis and its progression.

Methods: In this case-control study, we enrolled 66 subjects with chronic hepatitis C (CHC) with early stage of fibrosis and 65 subjects with $\mathrm{CHC}$ with late-stage fibrosis. Also, 40 subjects were included as normal controls. The six main miRNAs, i.e., miR-138, miR-140, miR-143, miR-325, miR-328, and miR-349, were measured using the reverse transcription-polymerase chain reaction.

Results: In the cases of $\mathrm{CHC}$ both with early and late stage of fibrosis, the circulating levels of the six main miRNAs were significantly higher than the levels in the control group. ROC analysis indicated that the sensitivity and specificity of miR-138 were $89.3 \%$ and $71.43 \%$, respectively, in the early stage of fibrosis. In the late stage, the sensitivity and specificity of miR-138 were 89.3 and $93.02 \%$, respectively, whereas, for miR-143, they were 75.0 and $88.4 \%$, respectively.

Conclusions: Circulating miR-138 could serve as a non-invasive biomarker for the detection of early fibrosis. Also, miR-138 and miR-143 could be specific biomarkers for indicating the late stage of liver fibrosis.

Keywords: liver fibrosis, microRNAs, chronic hepatitis C, hepatic stellate cell
\end{abstract}

\section{Introduction}

A major cause of chronic liver disease is Hepatitis C virus (HCV), and about 200 million people are infected worldwide. HCV infection is sensed by multiple innate immune pathways, resulting in chronic liver fibrosis (1), which is a pathological condition. This condition is caused by liver damage that leads to the excess accumulation of abnormal extracellular matrix (ECM) proteins, which is accompanied by the loss of liver function (2). Liver fibrosis is induced by hepatic stellate cells (HSCs), which are the major mesenchymal cells in the liver (3). Activated HSCs lose their lipid droplets, migrate to injured sites, and are transformed into myofibroblast-like cells. These cells secrete large amounts of ECM proteins, including collagen, laminin, proteoglycan, and fibronectin $(4,5)$. HSC is regulated by multiple signal transduction pathways, and its activation is considered to be a key event, making proteins from the pathways involved targets for micro RNAs (miRNAs) (6). Previous studies have shown that miRNAs in the liver play a fundamental role in the proliferation, differentiation, apoptosis, and migration of HSCs,

\section{Corresponding author:}

Professor Dr. Eman G. El-Ahwany, Immunology Department, Theodor Bilharz Research Institute, P.O. BOX: 30 Imbaba, Giza, Egypt. Tel: +20.1002050805, E-mail: ahwany@aucegypt.edu Received: November 12, 2015, Accepted: Decemebr 28, 2015, Published: January 2016 iThenticate screening: December 13, 2015, English editing: January 04, 2016, Quality control: January 06,2016 (C) 2015 The Authors. This is an open access article under the terms of the Creative Commons Attribution-NonCommercialNoDerivs License, which permits use and distribution in any medium, provided the original work is properly cited, the use is non-commercial and no modifications or adaptations are made. 
and the aberrant miRNA expression is related to liver fibrosis $(4,7,8)$. Also, it was shown that, during hepatic fibrogenesis, certain miRNAs integrate pro-fibrogenic and pro-inflammatory signals in HSCs, thereby controlling the expression of various extracellular matrix genes $(1,9)$. MiRNAs consist of approximately 22 nucleotides noncoding RNAs that participate in the post-transcriptional regulation of many protein-coding mRNA molecules. They do so by binding specifically with the 3-untranslated region (3-UTR) of the target gene. MiRNA inhibits the target expression either by mRNA degradation or translational repression $(10,11)$. The role of miRNAs that seems to be emerging in innate and adaptive immunity provides a strong indication of an association with the regulation of inflammatory diseases (5). In order to understand the critical pathways of HSC activation, recent studies performed comprehensive comparative bioinformatics analysis of microarrays of quiescent and activated HSC, and they identified 12 up-regulated miRNAs (miR-874, -290, -501,-349, -325-sp, -328, -138, -143, -207, -87, -140, -193) and nine downregulated miRNAs (miR-341, -206-3p, -156, -16, -375, -122, -146a, -29b, -126) $(6,8,12)$. These studies suggested that the altered miRNA patterns that occur after chronic liver disease have a significant effect on the progression of fibrosis. This is due to their potential to activate HSC, target the expression of ECM proteins, and the synthesis of mediators of the profibrogenic pathway (13). Although an important therapeutic advancement was achieved with the recent discovery of potent, direct-acting antiviral agents (DAAs) against HCV, even the trace amounts of HCV RNA from successfully-treated patients can be infectious $(1,14,15)$. Thus, there is a need for early diagnosis and therapeutic modalities for the prevention of HCV-related, end-stage liver disease. Based on recent studies, miRNAs have become the focus of interest as diagnostic biomarkers and therapeutic targets. However, since miRNAs are released into the bloodstream, it has been suggested that circulating miRNAs patterns hold great potential for non-invasive, blood-based biomarkers for serving as valuable prognostic indicators of the progression of fibrosis of the liver (16). In the current study, we explored the potential value of circulating miRNAs as noninvasive, predictive biomarkers for $\mathrm{HCV}$-induced fibrosis of the liver.

\section{Material and Methods}

\subsection{Study design and patients}

This study included 66 subjects with chronic hepatitis $\mathrm{C}(\mathrm{CHC})$ and early-stage fibrosis and 65 subjects with $\mathrm{CHC}$ with late-stage fibrosis who were admitted to the Department of Hepato-gastroenterology. Also, 40 subjects were included as healthy controls. Laboratory investigations included urine and stool analyses, liver function tests, alanine aminotransferase (ALT), aspartate amino-transferase (AST), prothrombin time, albumin, and bilirubin. Also, ultrasonography and clinical investigations were done for all of the subjects in the study. Six main miRNAs, i.e., miRNA-138, miRNA-140, miRNA-143, miRNA-325, miRNA-328, and miRNA-349, were selected based on the results of previous studies that demonstrated their notable expression pattern during the development of fibrosis of the liver. The six miRNAs were measured using Reverse Transcription (RT) and real-time reverse transcriptionpolymerase chain reaction (qPCR). All patients gave informed consent prior to entering the study in conformance with the guidelines of the 1975 Declaration of Helsinki, as reflected by approval of the institution's Human Research Ethics Committee.

\subsection{Laboratory investigations}

We conducted tests of liver functions and conducted serological diagnosis of viral hepatitis to determine the presence of chronic HCV infection. For more than six months, the subjects were administered reactive anti-HCV antibodies (using the Murex enzyme immunoassay kit, Dartford, England), and positive HCV-RNA was detected using the Abbott Real time HCV assay (Abbott Laboratories, Illinois, U.S.A.). Patients who had been treated previously for hepatitis $\mathrm{C}$ were excluded.

\subsection{Histopathologic Study}

We conducted an assessment of the grade of inflammation and the stage of fibrosis in 5- $\mu$ m-thick serial sections of formalin-fixed, paraffin-embedded blocks, which were stained with hematoxylin/eosin and Masson trichrome. The Metavir scoring system (The French METAVIR Cooperative Study Group) was used to determine the stage of hepatic fibrosis. Accordingly, the subjects were divided into groups of 36 patients who had F1 and F2 (early-stage fibrosis) and 35 patients who had F3 and F4 (late-stage fibrosis).

\subsection{RNA Extraction, Reverse Transcription (RT) and Quantitative PCR (qPCR)}

RNA was isolated from the serum of HCV patients using a Qiagen Viral RNA kit (Hilden, Germany). To evaluate the expression of the chosen miRNAs from the serum samples, we used RT and qPCR kits that were made specifically for accurate miRNA analysis (Applied Biosystems, USA). We performed the 15- $\mu \mathrm{L}$ RT reactions by using a TaqMan ${ }^{\circledR}$ microRNA Reverse Transcription Kit (Applied Biosystems, USA). We calculated the relative 
expression of miRNA using the comparative cycle threshold (CT) $\left(2^{-\Delta \Delta C T}\right)$ method (17), and the data were normalized using miRNA-16 as the endogenous control.

\subsection{Statistical Analysis}

The results data were log-transformed for analysis because of the magnitude and range of the relative miRNA expression levels that were observed, and the data were presented as mean $\pm \mathrm{SE}$. We used the Kolmogorov-Smirnov test to confirm that there was no evidence against normality for the log transformed data. The analysis of variance (ANOVA test) was used to evaluate the differences in the chosen miRNAs between the subjects and the controls. We constructed receiver operating characteristic (ROC) curves, and the areas under the curves (AUCs) were calculated to evaluate the specificity and sensitivity for the subjects and the controls. All statistical tests were twosided, and a probability level of $\mathrm{p}<0.05$ was considered to be statistically significant. We analyzed the data using IBM-SPSS software, version 22.

\section{Results}

Clinical investigation and laboratory data of studied cases are provided in Table 1 . In this study, six up-regulated miRNAs were selected for validation by RT and qPCR, i.e., miR-138, miR-140, miR-143, miR-325, miR-328, and miR-349. We studied their expression levels by isolating miRNAs from the serum of 66 patients with early fibrosis, 65 patients with late fibrosis, and 40 healthy controls. Interestingly, the values of all examined miRNAs were increased significantly in early and late fibrosis compared to healthy control $(\mathrm{p}<0.001, \mathrm{p}<0.01$, and $\mathrm{p}<0.05$, respectively). MiR-138 showed the highest significant value in early and late fibrosis compared to healthy controls $(\mathrm{p}<0.001)$, followed by miR-140, and miR-143 in late fibrosis $(\mathrm{p}<0.001)$, while, in early fibrosis, the values were $(\mathrm{p}<0.01$ and $\mathrm{p}<0.05$, respectively, compared to the healthy controls. However, miR-328, miR-349, and miR-325 showed moderately significant increasing values in late fibrosis $(\mathrm{p}<0.01)$ and in early fibrosis $(\mathrm{p}<0.05)$ compared to the healthy controls. These results are provided in Table 2.

Table 1. Demographic and laboratory characterization of early and late fibrosis and control groups

\begin{tabular}{|l|l|l|l|}
\hline Parameters & Control $(\mathrm{n}=40)$ & $\begin{array}{l}\text { CHC with early } \\
\text { fibrosis }(\mathrm{n}=66)\end{array}$ & $\begin{array}{l}\text { CHC with late fibrosis } \\
(\mathrm{n}=65)\end{array}$ \\
\hline Age & $41.7 \pm 13.6$ & $49.3 \pm 7.6$ & $58.2 \pm 9.6$ \\
\hline Male/female ratio & $23 / 17$ & $39 / 27$ & $40 / 25$ \\
\hline Pallor & Absent & Mild/moderate & Severe \\
\hline Palmer erythema & Absent & Absent/mild & Moderate \\
\hline Spider naevi & Absent & Absent/mild & Moderate \\
\hline Edema of the lower limb & Absent & Absent/mild & Moderate \\
\hline Ascitis & Absent & Absent/minimal & Mild/moderate \\
\hline Encephalopathy & Absent & Absent & Absent/present \\
\hline Albumin $(\mathrm{g} / \mathrm{dl})$ & $4.1 \pm 0.9$ & $3.7 \pm 0.6$ & $2.6 \pm 0.2$ \\
\hline Bilirubin (mg/dl) & $<1$ & $1-3$ & $>3$ \\
\hline ALT (IU/L) & $36.9 \pm 5.2$ & $69.1 \pm 17.3$ & $57.8 \pm 15.3$ \\
\hline AST (IU/L) & $29.7 \pm 8.3$ & $52.8 \pm 13.9$ & $49.7 \pm 11.1$ \\
\hline Prothrombin time/ seconds & 1 & 1.6 & $>6$ \\
\hline
\end{tabular}

Data are expressed as mean \pm SD, ALT: Alanine amino transferase, AST: Aspartate amino transferase

Table 2. Real-time qPCR expression levels of miRNAs in serum of the different studied groups

\begin{tabular}{|l|l|l|l|}
\hline Groups & Control & Early Fibrosis & Late Fibrosis \\
\hline miR-138 & $50.3 \pm 1.15$ & $101.90 \pm 0.8^{* * *}$ & $160.89 \pm 0.70^{* * *}$ \\
\hline miR-140 & $24.30 \pm 0.69$ & $51.26 \pm 0.8^{* *}$ & $103.71 \pm 1.01^{* * *}$ \\
\hline miR-143 & $12.59 \pm 0.54$ & $35.87 \pm 0.91^{*}$ & $84.24 \pm 0.55^{* * *}$ \\
\hline miR-328 & $21.2 \pm 0.62$ & $29.61 \pm 0.67^{*}$ & $72.18 \pm 0.58^{* *}$ \\
\hline miR-325 & $12.97 \pm 0.72$ & $14.9 \pm 0.49^{*}$ & $64.53 \pm 0.58^{* *}$ \\
\hline miR-349 & $30.4 \pm 0.72$ & $34.70 \pm 0.48^{*}$ & $63.59 \pm 0.56^{* *}$ \\
\hline
\end{tabular}

Data shown are expressed as mean \pm S.E., ${ }^{* * *} p<0.001,{ }^{* *} p<0.01,{ }^{*} p<0.05$ 
Box plot diagrams of the expression of miR-138, miR-140, miR-143, miR-325, miR-328, and miR-349 in early and late fibrosis and in the healthy controls are shown in Figure 1. Correlation analysis was performed, and it was found that there was a negative correlation between miR-143 and miR-328 $(r=-0.311$ and $p<0.05)$, at the same time there was positive correlation between miR-140 and miR-328 ( $\mathrm{r}=0.526$ and $\mathrm{p}<0.01)$, indicating that the increasing values of miR-328 in the serum influenced the concentration of miR-140 in the serum. To further investigate the characteristics of miR 138 as a potential biomarker in early- and late-stage fibrosis of CHC, ROC curve analyses were performed. Analysis of the ROC curves for serum miR 138 demonstrated an AUC (area under the ROC curve) of 0.866 (95\% CI: $0.7560 .941 ; p<0.001)$ with $89.3 \%$ sensitivity and $71.43 \%$ specificity in discriminating chronic $\mathrm{HCV}$ infection from healthy controls at a cut off value of 52.3 (Figure 2). In late fibrosis, the analysis of the ROC curve showed that miR-138 and miR- 143, with areas under the curves of 0.924 and 0.682 , respectively, and $\mathrm{p}<$ 0.001 and $\mathrm{p}<0.05$, respectively, and its sensitivity $=89.3 \%$ and specificity $=75.0 \%$, and sensitivity $93.02 \%$ and specificity $88.4 \%$ with a cut-off value $=62.9$ (Figure 3 ).
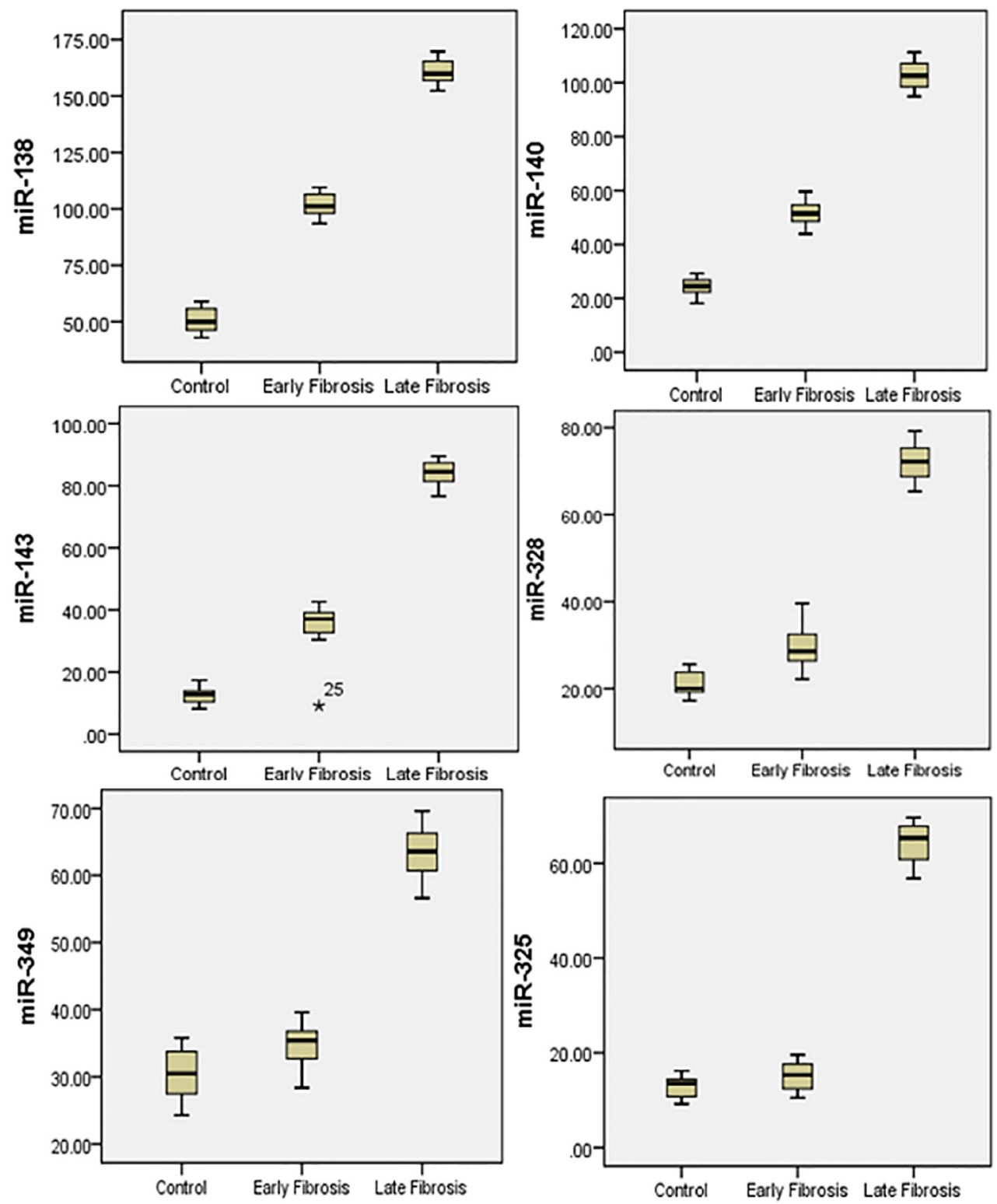

Figure 1. Box plot diagram of the expression of miRNAs in $\mathrm{HCV}$-induced liver fibrosis patients: The box indicates the $25^{\text {th }}$ and $75^{\text {th }}$ percentiles of the data, and the middle line indicates the median. A line extends from the minimum value to the maximum value, excluding outliers that are displayed as separate points. 


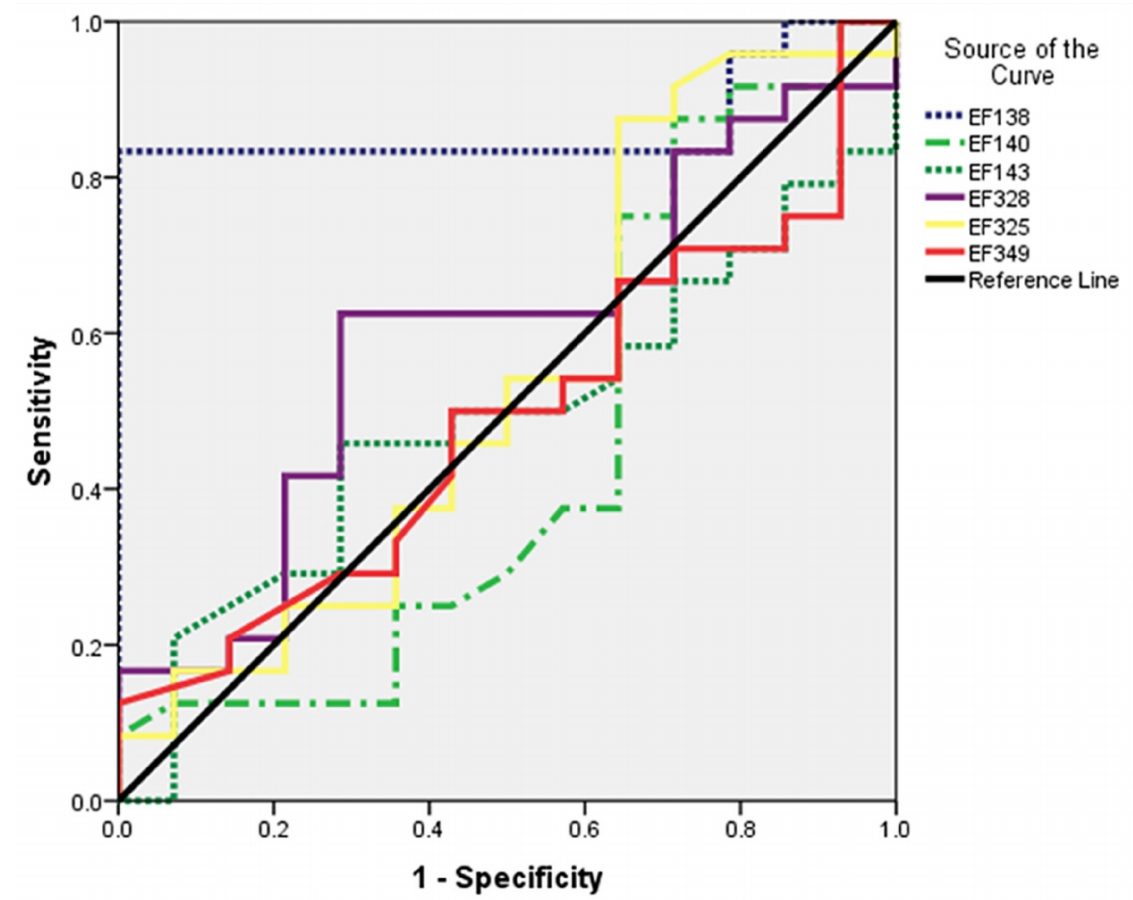

Figure 2. ROC curve analysis displaying the diagnostic power of the studied miRNAs in early stage of fibrosis

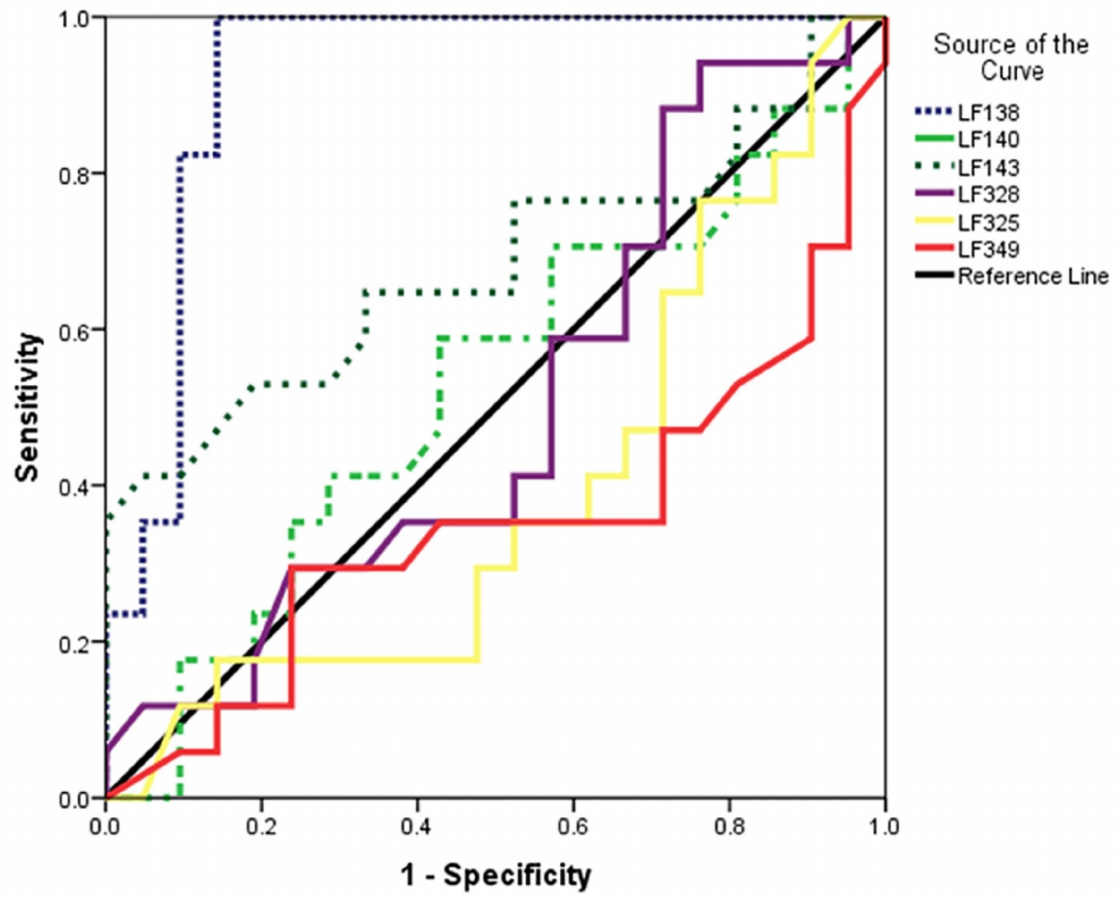

Figure 3. ROC curve analysis displaying diagnostic power of studied miRNAs in late stage of fibrosis

\section{Discussion}

Activated HSCs are the principal cell type that promotes the synthesis and deposition of ECM proteins in response to increased levels of circulating inflammatory signals derived from damaged parenchymal cells. So, HSCs activation serves as the critical step in the initiation and progression of liver fibrosis. In previous studies, in patients with chronic liver disease and liver fibrosis, array-based studies identified several miRNAs to be upregulated in serum compared to healthy controls, several of which also correlated with the degree of liver fibrosis (13). In this study, we measured six miRNAs (miR-349, -325, -328,-138, -143, -140), which are considered as upregulated 
miRNAs in activated HSCs. We measured these miRNAs in serum of patients with early and late fibrosis because they have been demonstrated to be very specific and stable in human serum and plasma $(11,18,19)$. Our results indicated that miR-138 and miR-140 were highly detected in early and late fibrosis compared to healthy patients and more than other miRNAs. This indicates the obvious role of these miRNAs in activation of HSCs and progression of liver fibrosis. Hepatocellular injury followed by inflammation and activation of the innate immune system may lead to early stage liver fibrosis, resulting in HSC activation (20). The transition process of HSC into a myofibroblastic cell type is accompanied by stimulation of a variety of profibrogenic growth factor profiles. At the same time, other targeted genes are those critical for HSC activation and proliferation, are markedly up-regulated during hepatic fibrogenesis. These targeted genes regulated by miRNAs include transforming growth factor-beta (TGF- $\beta$ ), the platelet-derived growth factors (PDGF), mitogen-activated protein kinase (MAPK), vascular endothelial growth factor (VEGF), and tissue inhibitor of metalloproteinase I (TIMPs) $(1,8)$. These targeted genes' signaling has been found to be essential for myofibroblastic transdifferentiation of HSCs and stimulates proliferation and the synthesis of type I collagen and matrix metalloproteinases, leading to increasing synthesis of ECM (10). Of these targeted genes, TGF- $\beta$ has been recognized as the most potent fibrogenic cytokine, which leads to secretion of an excessive amount of ECM components, predominantly type I collagen, that is deposited as fibers $(1,5,21)$. In advanced stages of liver fibrosis, bridging collagen bands occurs, and the liver may contain up to six times more ECM than normal (5), thereby compromising organ function and leading to its failure (1). Previous studies assumed that miRNAs may be involved in the altered gene expression profile of myofibroblastic HSC in early stage of fibrosis $(8,13,22)$. Also, miRNAs definitely contribute to the profibrogenic changes during fibrogenesis in late stage of fibrosis, not only by targeting ECM production, but also by interaction with the TGF- $\beta$ signaling pathway (13). Previous studies demonstrated that the activation of HSCs related to these miRNAs (miR-138, -140, -143) may regulate multiple functions especially inducing cell cycle arrest and apoptosis by targeting Bcl-2 gene and caspase-9 signaling pathway. However, they may play an important role in the interaction with the TGF- $\beta$ signaling pathway $(8,23)$. In this study, our findings were consistent with these reports; however, miR-138 had obvious increasing value in early fibrosis than the other studied miRNAs. Hyaluronan (HA) is a glycosaminoglycan synthesized by HSCs, and it is a component of ECM. The increased expression of HA and its cell-surface receptor (CD44) may reflect increased synthesis of ECM by HSCs. Recent work has found that miR-328 regulates the binding interaction of CD44 with its ligand hyaluronan (24). The 3'UTR of CD44 targeted by miR-328 resulted in regulating ECM synthesis by causing an increased expression of CD44, fibronectin, and type I collagen (23). This finding was consistent with our result that the increasing levels of miR-325, $-349,-328$ in late-stage liver fibrosis, more than in early-stage fibrosis, may reflect the role of these miRNAs in the progression of fibrosis by targeting the gene responsible for increasing ECM synthesis.

\section{Conclusions}

This study indicated that miRNAs might be considered potential diagnostic biomarkers for activation of HSCs and formation of fibrosis and offer insight into its progression. We found that miR-138 was the more specific miRNA expressed in early and late fibrosis, followed by miR-143 in late fibrosis. However, the other miRNAs that were studied (miR-140, miR-235, miR-238, miR-349) showed increasing expression in late stage of fibrosis more than in the early stage. Thus, it is recommended that further study be conducted at a larger scale.

\section{Acknowledgments:}

This work was supported from both the internal project No. 86 diagnosis, TBRI and the American University in Cairo (AUC).

\section{Conflict of Interest:}

There is no conflict of interest to be declared.

\section{Authors' contributions:}

All authors contributed to this project and article equally. All authors read and approved the final manuscript.

\section{References}

1) Kown Y, Ray RB, Ray R., "Hepatitis $C$ virus infection: establishment of chronicity and liver disease progression,” EXCLI Journal. 2014; 13: 977-96.

2) Kumar V and Mahato R. Delivery and targeting of miRNAs for treating liver fibrosis. Pharmaceutical Research, 2015; 32: 341-61. doi: 10.1007/s11095-014-1497-x, PMid: 25186440 
3) Friedman RC, Farh KK, Burge CB, Bartel DP. Most mammalian mRNAs are conserved targets of micro RNAs. Genome Res. 2009; 19: 92-105. doi: 10.1101/gr.082701.108, PMid: 18955434, PMCid: PMC2612969

4) He Y, Huang C, Zhang SP, Sun X, Long XR, Li J. The potential of micro RNAs in liver fibrosis. Cellular Signaling 2012; 24: 2268-72. doi: 10.1016/j.cellsig.2012.07.023, PMid: 22884954

5) Roy S, Benz F, Luedde $T$ and Roderburg C. The role of miRNAs in the regulation of inflammatory processes during hepatofibrogenesis. Hepatobiliary 2015; 4(1): 24-33.

6) Wang B, Li W, Guo K, Xiao Y, Wang Y, Fan J. MiR-181b promotes hepatic stellate cells proliferation by targeting p 27 and is elevated in the serum of cirrhosis patients. Biochemical and Biophysiol research Communications 2012; 421: 4-8. doi: 10.1016/j.bbrc.2012.03.025, PMid: 22446332

7) Chen C, Zhang Y, Zhang L, Weakley SM, Yao Q. Micro RNA-196 critical roles and clinical applications in development and cancer, J Cell Mol Med. 2011;15: 14-23. doi: 10.1111/j.1582-4934.2010.01219.x, PMid: 21091634, PMCid: PMC3276076

8) Guo CJ, Pan Q, Cheng T, Jiang B, Chen GY and Li DG. Changes in miRNAs associated with hepatic stellate cell activation status identify signaling pathways. FEBS Journal 2009; 276: 5163-73. doi: 10.1111/j.1742-4658.2009.07213.x, PMid: 19674103

9) Roberts AP, Lewis AP, Jopling CL. The role of microRNAs in viral infection. Prog Mol Biol Transl Sci 2011; 102: 101-39. doi: 10.1016/B978-0-12-415795-8.00002-7, PMid: 21846570

10) Mendias CL, Gumucio JP and Lynch EB. Mechanical loading and TGF- $\beta$ change the expression of multiple miRNAs in tendon fibroblasts. J Appl Physiol 2012; 113(1): 56-62. doi: 10.1152/japplphysiol.00301.2012, PMid: 22539168, PMCid: PMC3404830

11) Shrivastava S, Mukherjee A \& Ray RB. Hepatitis C virus infection, micro RNA and liver disease progession. World J Hepatol 2013; 5 (9): 479-88. doi: 10.4254/wjh.v5.i9.479, PMid: 24073299, PMCid: PMC3782685

12) Murakami Y, Toyoda H, Tanaka M, Kuroda M, Harado Y, Matsuda F, et al. The progression of liver fibrosis is related with overexpression of the miR-199 and 200 families. PLOS One 2011; 6: 16081-95. doi: 10.1371/journal.pone.0016081, PMid: 21283674, PMCid: PMC3025920

13) Huang J, Yu X, Fries JWU, Zhang L and Odenthal M. microRNA function in the profibrogenic interplay upon liver disease. Int J Mol Sci 2014; 15(6); 9360-71. doi: 10.3390/ijms15069360, PMid: 24871365, PMCid: PMC4100099

14) Au JS, Pockros PJ. Novel therapeutic approaches for hepatitis C. Clin Pharmacol Ther 2014; 95: 78-88. doi: 10.1038/clpt.2013.206, PMid: 24126682

15) Veerapu NS, Park SH, Tully DC, Allen TM, Rehermann B. Trace amounts of sporadically reappearing HCV RNA can cause infection. J Clin Invest 2014; 124: 3469-78. doi: 10.1172/JCI73104, PMid: 25003189, PMCid: PMC4109550

16) Chang $\mathrm{C}$, Lin $\mathrm{C}$, Hsieh W, Lai H, Tsai $\mathrm{C}$ and Cheng Y. microRNA expression profiling in PBMCs: A potential diagnostic biomarker of chronic hepatitis C. Disease markers 2014; 1-9. doi: 10.1155/2014/367157, PMid: 25505813, PMCid: PMC4255053

17) Schmittgen TD, Livak KJ. Analyzing real-time PCR data by the comparative C(T) method. Nat Protoc 2008; 3: 1101-8. doi: 10.1038/nprot.2008.73

18) Brase JC, Wuttig D, Kuner R, Sultmann H. Serum micro RNAs as non-invasive biomarkers for cancer. Mol cancer, 2010; 9: 306-11. doi: 10.1186/1476-4598-9-306, PMid: 21110877, PMCid: PMC3002336

19) Allergra A, Alonic A, Campo S, Penna G, Petrungaro A, et al. Circulating micro RNAs; new biomarkers in diagnosis, prognosis and treatment of cancer (review). Int J Oncol 2012; 41: 1897-1912.

20) Hernandez-Gea V. and Friedman SL. Pathogenesis of liver fibrosis. Annu Rev Pathol 2011; 6: 425-56. doi: 10.1146/annurev-pathol-011110-130246, PMid: 21073339

21) Schrum LW and Lakner AM. Treatment of fibrosis using microRNA 19b. U.S. patent 2014; 8: 1-17.

22) Ji J, Zhang J, Huang G, Qian J, Wang X, Mei S. Over-expressed microRNA-27a and -27b influence that accumulation and cell proliferation during rat hepatic stellate cell activation. FEBS Lett 2009; 583: $759-66$. doi: 10.1016/j.febslet.2009.01.034, PMid: 19185571

23) Li J, Ghazwani M, Zhang Y. miR-122 regulates collagen production via targeting hepatic stellate cell migration and activation. Exp Cell Res 2013; 317: 1714-25. doi: 10.3389/fimmu.2012.00116, PMid: 22593760, PMCid: PMC3349963

24) Liu Y, Munker S, Mullenbach R. IL-13 signaling in liver fibrogenesis. Front Immunol 2012; 3: 116-8. 